\title{
Fosnetupitant/Palonosetron Hydrochloride
}

National Cancer Institute

\section{Source}

National Cancer Institute. Fosnetupitant/Palonosetron Hydrochloride. NCI Thesaurus.

Code C146720.

A combination formulation containing the prodrug of netupitant, fosnetupitant, a selective neurokinin 1 receptor (NK1R; TACR1) antagonist, and the hydrochloride salt form of palonosetron, a selective serotonin (5-hydroxytrytamine; 5-HT) receptor subtype 3 (5-HT3R) antagonist, with potential antiemetic activity. Upon intravenous administration, fosnetupitant is converted by phosphatases to its active form netupitant. Netupitant competitively binds to and blocks the activity of NK1Rs in the central nervous system (CNS), by inhibiting binding of the endogenous tachykinin-derived neuropeptide substance P (SP) to NK1R. This prevents delayed emesis, which is associated with SP secretion. Palonosetron competitively blocks the interaction of 5-HT with 5-HT3Rs located on vagal afferent nerves in the chemoreceptor trigger zone (CTZ). This inhibits acute emesis associated with 5-HT secretion and subsequent 5-HT3R activation.

Altogether, this results in inhibition of acute and delayed nausea and vomiting, and may prevent chemotherapy-induced nausea and vomiting (CINV). 